\title{
Diffuse alveolar hemorrhage (DAH) syndrome: A rare complication of anticoagulant therapy in a patient with Waldenstrom macroglobulinemia
}

\author{
Massimo Bolognesi ${ }^{1}$ and Giampiero Pasini ${ }^{2}$ \\ ${ }^{1}$ General Practice Medicine - ASL 112 District of Cesena via Ungaretti 49447521 Cesena, Italy \\ ${ }^{2}$ Director of Internal Medicine Department - M. Bufalini Hospital, Via le Ghirotti, 28647521 Cesena, Italy
}

\begin{abstract}
Diffuse Alveolar Hemorrhage (DAH) is a clinical syndrome characterized by hemoptysis, anemia, hypoxemic respiratory failure and diffuse pulmonary infiltrates. Various diseases are associated with the development of DAH syndrome. DAH is a rare event, however, it may be complicated by warfarin therapy, and it may have a worsening clinical course and can be a life-threatening condition. Here the authors report the case of a 76-year-old male, affected by Waldenstrom Macroglobulinemia (WM), who was admitted to hospital for cough, dyspnea, and hemoptysis. Chest X-ray and computed tomography revealed diffuse pulmonary consolidations, and bronchoalveolar lavage demonstrated diffuse alveolar hemorrhage. After ten days of appropriate therapy with corticosteroids, antibiotics, and crystalloid intravenous fluid, the patient returned in good status.
\end{abstract}

\section{Introduction}

Diffuse alveolar hemorrhage (DAH) concerns a clinical syndrome, characterized by extensive bleeding in the acinar portion of the lung [1].

Bleeding into the alveolar spaces of the lungs characterizes the syndrome and is due to disruption of the alveolar-capillary basement membrane. This disruption is caused by injury or inflammation of the arterioles, venules, or alveolar septal (alveolar wall or interstitial) capillaries.

It usually presents with clinical features of hemoptysis, anemia, diffuse radiographic alveolar consolidation, and sometimes acute respiratory failure [2].

The specific pathophysiology and manifestations vary depending on the cause. For example, isolated pauci-immune pulmonary capillaritis is a small-vessel vasculitis limited to the lungs; its only manifestation is alveolar hemorrhage affecting people aged 18 to $35 \mathrm{yr}$.

Idiopathic pulmonary hemosiderosis is diffuse alveolar hemorrhage with no detectable underlying disorder; it occurs mainly in children $<10 \mathrm{yr}$ and is thought to be due to a defect in the alveolar capillary endothelium, possibly due to autoimmune injury.

DAH is caused by various pathologies that damage the alveolar- capillary barrier or by disorders of coagulation. These underlying diseases include lung infections, pulmonary embolism, Wegener's granulomatosis, Goodpasture's syndrome, systemic lupus erythematosus and Behçet's syndrome [3].

Immunoglobulin $\mathrm{M}$ (IgM) associated disorders, such as cryoglobulinemia and Waldenstrom Macroglobulinemia (WM), are caused by autoreactivity of IgM paraproteins. Cryoglobulinemia is usually classified into three types according to Brouet's classification.
Type 1 is characterized by the presence of a monoclonal immunoglobulin which can be seen in lymphoproliferative disorders such as chronic lymphatic leukemia, multiple myeloma or non-Hodgkin lymphoma. Type 2 has a monoclonal component (mostly IgM) and polyclonal immunoglobulin which can lead to immune-complex vasculitis. Type 3 has polyclonal immunoglobulins of different isotypes. Types 2 and 3 are also called mixed type cryoglobulinemia [4]. Cryoglobulinemia should be considered in small vessel vasculitis. As literature report [5], $\mathrm{DAH}$ is a rare complication of type 2 cryoglobulinemia.

Only a few reports have mentioned the manifestation of alveolar hemorrhage in patients receiving anticoagulants, such as warfarin and heparin [6].

Furthermore, DAH complicated by warfarin therapy is associated with a high mortality rate [7].

Patients with alveolar hemorrhage present with complaints of cough, dyspnea, and hemoptysis.

It is crucial to understand that even the most severe cases of alveolar hemorrhage may not cause hemoptysis. In fact, one-third of the patients will not have hemoptysis, despite active intra-alveolar bleeding [8].

\section{Case report}

Here the authors describe the case of a 76-year-old male who was admitted to the hospital's internal medicine department for

Correspondence to: Massimo Bolognesi, General Practice Medicine - ASL 112 District of Cesena Via Ungaretti 49447521 Cesena, Italy, E-mail: massbolo1@tin.it

Key words: Diffuse alveolar hemorrhage, Anticoagulant therapy, Waldenstrom macroglobulinemia

Received: May 08, 2015; Accepted: June 19, 2015; Published: June 23, 2015 
cough, dyspnea, and hemoptysis. His clinical history was significant for Waldenstrom Macroglobulinemia (WM, also known as lymphoplasmacytic lymphoma), restrictive cardiomyopathy due to amyloidosis, and permanent atrial fibrillation. He had undergone prophylactic anticoagulation therapy with warfarin for many years. The physical examination showed a feverish patient, pale, coughing and spitting blood. Pulmonary auscultation revealed crackles and an irregular heart rhythm was present, with mild peripheral edema.

The Chest $\mathrm{x}$-ray (Figure 1) showed diffuse bilateral alveolar infiltrates suggestive for diffuses alveolar hemorrhage. Subsequent CT of the chest and bronchoscopy with bronchoalveolar lavage (BAL) was made to confirm the diagnosis. Bronchoscopy showed fresh blood, on the whole, bronchial system while specimens showed blood with numerous erythrocytes and a CD8+ T-lymphocyte alveolitis with an accumulation of T-cells; lavage fluid typically remains hemorrhagic or becomes increasingly hemorrhagic after sequential sampling. No openlung biopsy was made.

Chest computed tomography (Figure 2) showed newly developed extensive ground-glass attenuations with crazy-paving appearance on the entire lung fields, typical of DAH findings.

All cultures of sputum, BAL fluid and blood did not reveal any causative pathogens. The patient showed pure DAH confined to the lungs, and there was no evidence of disseminated intravascular coagulation or hemorrhagic events occurring elsewhere in the body. Urinalysis, INR and serum creatinine levels were within a normal range. Anti-DNA/ANCA/Anti-GBM serology was negative.

A moderate normocytic anemia and elevated erythrocyte sedimentation rate (ESR) were present.

Clarithromycin (500 mg, twice daily) and Meropenem (1000 mg,

\section{Chest X-ray - Diffuse Alveolar Infiltrates}

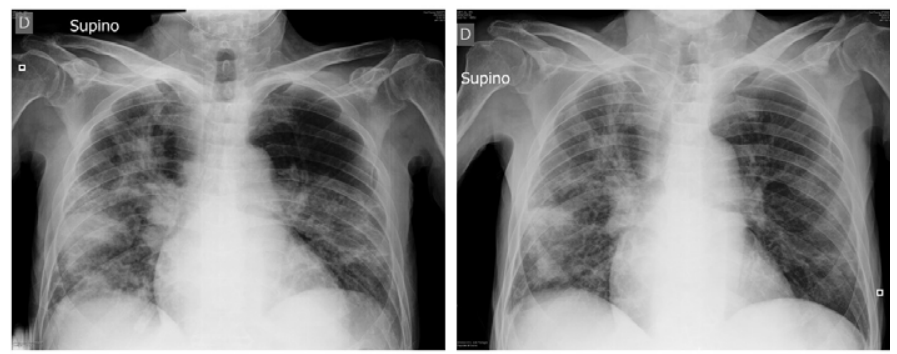

Figure 1. Chest X-ray shows diffuse bilateral alveolar infiltrates.

\section{Chest CT - Diffuse Alveolar Hemorrhage}

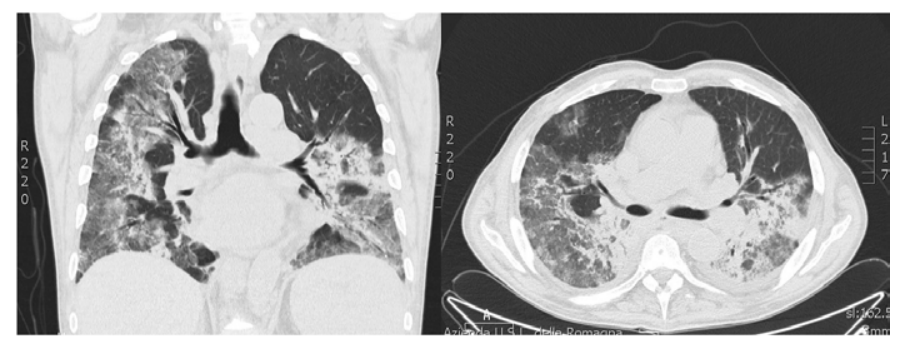

Figure 2. Chest $\mathrm{CT}$ shows extensive ground-glass attenuations with crazy-paving appearance on whole lung fields. once daily) were empirically administered to prevent pneumonia, associated with a high dose of prednisone. Warfarin was discontinued. After a ten days treatment, the patient returned in good condition.

\section{Discussion}

DAH refers to the accumulation of intra-alveolar red blood cells originating from the alveolar capillaries1. Hemoptysis is the usual presenting symptom; however it is not always present, even when hemorrhage is severe [3].

As it is well known, DAH may occur in several pathologic conditions, including various vasculitis, autoimmune diseases, acute post-streptococcal glomerulonephritis, and potentially any pneumorenal syndrome. Other rare causes, such as drugs, inhaled cocaine, diphenyl-hydantoin, sirolimus, leflunomide, and everolimus, can also lead to DAH [9].

The underlying cause of DAH is generally reflected in the histopathologic pattern. One of the three different histopathologic patterns may be seen: pulmonary capillaritis, bland pulmonary hemorrhage, and diffuse alveolar damage [10].

However, even the role of open-lung biopsy is not well established and is generally not helpful in determining the underlying etiology of the DAH [8]. All anticoagulant medications have been associated with $\mathrm{DAH}$, including warfarin (Coumadin) and its derivatives, thrombolytic, agents targeting the platelet glycoprotein IIb/IIIa, and clopidogrel.

Warfarin is the most frequently prescribed oral anticoagulant inhibiting vitamin $\mathrm{K}$ utilization by the liver cells. Systemic bleeding events can occur in any organ or tissue, including the brain, and gastrointestinal or genitourinary tracts. Nevertheless, the rate of alveolar hemorrhage in patients taking anticoagulant therapy is low and forms a minor part of bleeding complications [11].

DAH is a rare complication of warfarin therapy. Since the first case of DAH caused by warfarin intoxication reported by Brown et al. [12] in 1965, few other reports have been described in the literature [13]. Warfarin-induced DAH is usually severe and can be lethal [14].

Here the authors wish to make some remarks related to this case. Firstly, it is very important to confirm an early diagnosis in clinically suspected patients with DAH since it can be lethal due to anemia and respiratory failure. BAL is the gold standard for the diagnosis, and progressively hemorrhagic BAL finding in serial samples or positive iron studies of BAL will confirm the diagnosis.

However, a careful approach is needed as bronchoscopy itself can cause massive pulmonary bleeding. Secondly, here the serum types of cryoglobulins were not analyzed. Therefore, a vasculitis from mixed cryoglobulinemia in a patient with long-standing Waldenstrom's macroglobulinemia secreting IGM cannot be excluded as a possible etiology.

Indeed, a lung biopsy could have found aspects of typical pulmonary capillaritis to make a more precise diagnosis. Unfortunately, a lung biopsy was not performed, so, this remains a clinical hypothesis.

Moreover, the incidence of anticoagulants-induced DAH may increase in future, because of the growth of an elderly population with comorbidities such as Waldenstrom macroglobulinemia, who need anticoagulants due to conditions such as atrial fibrillation, cardiovascular diseases, and valvular heart diseases. 


\section{Conclusion}

Although patients with DAH may not always appear critically ill, the diagnosis should be made quickly and the patient treated as a medical emergency. The first goal is to well establish the diagnosis. A detailed history can often help establish the risk factors and most likely the etiology of the alveolar hemorrhage. This case report provides a further contribution to the knowledge of the topic.

\section{Competing interests}

The authors declare they have no competing interests.

\section{References}

1. Lara AR, Schwarz MI (2010) Diffuse alveolar hemorrhage. Chest 137: 1-8.

2. Collard HR, Schwarz MI (2004) Diffuse alveolar hemorrhage. Clin Chest Med 25: 583-592, vii. [Crossref]

3. Lara AR, Frankel SK, Schwarz MI. Diffuse alveolar hemorrhage. In: Interstitial Lung Disease, (5thedn), Schwarz MI, King TE Jr (Eds), People's Medical Publishing House, Shelton, CT, USA 2011. p.805

4. Brouet JC, Clauvel JP, Danon F, Klein M, Seligmann M (1974) Biologic and clinical significance of cryoglobulins. A report of 86 cases. Am J Med 57: 775-788. [Crossref]

5. Schilders J, Brouwers A, Van Zaanen H (2013) Cryoglobulinemia complicated by diffuse alveolar hemorrhage. Neth J Crit Care 17: 15-18.
6. Santalo M, Domingo P, Fontcuberta J, Franco M, Nolla J (1986) Diffuse pulmonary hemorrhage associated with anticoagulant therapy. Eur J Respir Dis 69: 114-119. [Crossref]

7. Waness A, Aldabbagh T, Harakati M (2009) Diffuse alveolar haemorrhage secondary to warfarin therapy for atrial fibrillation: a case report and literature review. BMJ Case Rep 2009. [Crossref]

8. Zamora MR, Warner ML, Tuder R (1997) Diffuse alveolar hemorrhage and systemic lupus erythematosus. Clinical presentation, histology, survival, and outcome. Medicine (Baltimore) 76: 192-202. [Crossref]

9. Vandewiele B, Vandecasteele SJ, Vanwalleghem L, De Vriese AS (2010) Diffuse alveolar hemorrhage induced by everolimus. Chest 137: 456-459. [Crossref]

10. Travis WD, Colby TV, Lombard C, Carpenter HA (1990) A clinicopathologic study of 34 cases of diffuse pulmonary hemorrhage with lung biopsy confirmation. Am J Surg Pathol 14: 1112-1125. [Crossref]

11. Coon WW, Willis PW 3rd (1974) Hemorrhagic complications of anticoagulant therapy. Arch Intern Med 133: 386-392. [Crossref]

12. Brown OL, Garvey JM, Stern CA (1965) Roentgenogram of the month. Dis Chest 48 525-526. [Crossref]

13. Erdogan D, Kocaman O, Oflaz H, Goren T (2004) Alveolar hemorrhage associated with warfarin therapy: a case report and literature review. Int $J$ Cardiovasc Imaging 20: 155-159. [Crossref]

14. Thomas JK, Jayaprakash K, Misiriya KJ, Khadar SA, Pappachan JM (2008) Catastrophic pulmonary alveolar hemorrhage complicating warfarin therapy. $J$ Assoc Physicians India 56: 34. [Crossref]

Copyright: (C2015 Bolognesi M. This is an open-access article distributed under the terms of the Creative Commons Attribution License, which permits unrestricted use, distribution, and reproduction in any medium, provided the original author and source are credited. 